RECYT

Año 22 / № 33 / 2020 / 85-93

\title{
Películas y materiales compuestos basados en almidón de mandioca acetilado de alto grado de sustitución
}

\section{Films and composites based on acetylated cassava starch of high degree of substitution}

\author{
Pamela S. Cuenca ${ }^{1, *}$, Laura A. Ramallo ${ }^{1}$, Oscar A. Albani ${ }^{1}$ \\ 1- Laboratorio de Preservación y Envases. Instituto de Materiales de Misiones \\ UNaM-CONICET, Félix de Azara 1552, CP: N3300LQD, Misiones, Argentina. \\ *E-mail: pamcuenca@fceqyn.unam.edu.ar; pamcuenca@gmail.com \\ Recibido el 10 de septiembre de 2019, Aprobado el 20 de enero de 2020.
}

\section{Resumen}

\begin{abstract}
En este trabajo se desarrollaron películas de acetato de almidón de mandioca (AAM) de alto grado de sustitución ( $\mathrm{DS}=2,9)$ y materiales compuestos consistentes de la resina AAM soportada en papel Kraft. Como un paso previo, la síntesis del acetato de alto grado fue llevada a cabo empleando ácido metilsulfónico como catalizador. Se realizó la caracterización de las propiedades mecánicas y de barrera de las películas obtenidas. Las películas de almidón de mandioca acetilado resultaron homogéneas y continuas, de apariencia plástica, pero frágiles. El agregado de $50 \%$ de plastificante permitió obtener películas con buenas propiedades mecánicas (tensión de rotura 17,5 MPa y deformación de rotura de $8,2 \%$ ) pero que, con el almacenamiento, se volvieron cerosas y frágiles. Las películas compuestas de papel Kraft - acetato de almidón de mandioca tienen valores de permeabilidad al vapor de agua menores (hasta el 51\%) que los papeles sin tratar, a la vez que sus propiedades mecánicas son mejores (tracción de rotura 33 - 42 MPa y elongación 2 - 4\%). Por lo tanto, el desarrollo de envases biodegradables basados en materiales de almidón acetilado - celulosa parece una buena opción para el reemplazo de envases que no son ambientalmente compatibles.
\end{abstract}

Palabras clave: Acetato de almidón de mandioca; Alto grado de sustitución; Películas libres; Materiales compuestos.

\section{Abstract}

In this work, Cassava starch acetate $(\mathrm{CSA})$ with a high degree of substitution films (DS $=2.9)$ and composite materials consisting of CSA resin supported on Kraft paper were developed.

As a previous step, the synthesis of high degree acetate was carried out by using methylsulfonic acid as a catalyst. The characterization of the mechanical and barrier properties of the films obtained was performed. Acetylated cassava starch films were homogeneous and continuous, with plastic appearance, but brittle. The addition of $50 \%$ plasticizer allowed to obtain films with good mechanical properties (tensile strength at break 17,5 MPa and deformation $8,2 \%$ ) but with storage, they became waxy and brittle. The films composed of Kraft paper cassava starch acetate - have lower water vapor permeability values (up to $51 \%$ ) than untreated papers, and their mechanical properties are better (tensile strength at break 33- $42 \mathrm{MPa}$ and deformation 2 - 4\%). Therefore, the development of biodegradable packages based on acetylated starch-cellulose materials seems a good option for the replacement of packages that are not environmentally compatible.

Keywords: Cassava starch acetate; High degree of substitution; Free films; Composites.

Introducción

La contaminación del ambiente debida a los desechos de envases plásticos es uno de los problemas a resolver por la sociedad actual. En los últimos años, el interés en encontrar alternativas ecológicas para el material plástico no degradable hecho de petróleo, ha llevado a muchos investigadores a poner énfasis en los materiales poliméricos naturales de origen agrícola [1-4].
El almidón ha sido una de las mejores alternativas porque es un material renovable de bajo costo. Entre los almidones, el de mandioca destaca por su bajo precio y amplia disponibilidad.

El almidón presenta el inconveniente que, en su estado nativo, es muy sensible a la humedad y sus películas tienen alta permeabilidad al vapor de agua. Esto ha llevado a un importante numero de investigadores a trabajar con modificaciones del almidón. De las distintas modificaciones 
químicas posibles, la acetilación, es una de las formas más simples de mejorar las propiedades fisicoquímicas y funcionales del almidón [5].

Numerosos investigadores estudiaron el efecto de la acetilación en las propiedades de los acetatos de bajo y medio grado de sustitución [6] [7] [8] [9] [10]. Todas las modificaciones realizadas en los trabajos antes citados conducen a un material con buenas propiedades mecánicas pero soluble en agua.

Acetatos con alto grado de sustitución han sido preparados utilizando distintos procedimientos en medio alcalino [11]efficiently and with fewer byproducts. The method selected was to heat mixtures of dry corn starch, glacial acetic acid and anhydrides under pressure in small $(60 \mu \mathrm{l}$ [12] y en medio ácido [13] [14]. El método de acetilación propuesto por Feuer [13], utilizando como catalizador ácido metilsulfónico (MSA), provee un éster de almidón caracterizado por mayor peso molecular y menor pérdida de blancura, lo que sería útil en su uso como material de recubrimiento.

La hidrofobicidad del acetato de almidón se incrementa a medida que aumenta el grado de sustitución y la longitud de la cadena del sustituyente. Fringant y col. [15] informaron que acetato de almidón con un DS mayor que 1,7 presenta propiedades típicamente hidrofóbicas. Una serie de estudios encontraron buena compatibilidad de acetato de almidón con otros materiales, dando como resultado un material con propiedades mecánicas mejoradas [12] [16] [4].

Tarvainen y col. [17] mostraron que el acetato de almidón de papa de grado de sustitución 2.8, permite obtener por casting películas claras y continuas con alta hidrofobicidad. Tarvainen y col. [18] (SA investigaron la capacidad de los n-alquenil anhídridos succínicos o (n-ASA) como plastificantes externos, a fin de mejorar las características de formación de película de acetato de almidón de papa de grado de sustitución 2,8, obteniendo buenos resultados.

Por otro lado, considerando que existen pocos trabajos que describen el comportamiento de películas de acetato de almidón de alto grado y asumiendo que su carácter hidrófobo mejoraría las propiedades de barrera al vapor de agua, podrían utilizarse soluciones filmogénicas de este polímero como resina para generar materiales compuestos sobre una base de algún material de refuerzo como el papel Kraft, que contribuya a mejorar las propiedades mecánicas. Larotonda y col. [19] [20] mostraron que la impregnación de papeles simples con acetato de almidón de mandioca, con grado de sustitución de 1,4 mejora las propiedades de permeabilidad al vapor de agua, así como otras propiedades mecánicas del papel. Sin embargo, un DS $=1,41$ (el obtenido en estos trabajos) está en la zona de valores de DS en los que el acetilado de almidón es parcialmente soluble en agua.

El objetivo del presente trabajo fue sintetizar acetato de almidón de alto grado de sustitución (DS=2,9) y a partir de él, desarrollar películas libres hidrófobas y materiales compuestos consistentes en una resina de acetato de almidón de alto grado soportada en papel Kraft, a fin de obtener materiales aptos para envases biodegradables. Se realizó la caracterización de las propiedades mecánicas y de barrera al vapor de agua de los materiales obtenidos.

\section{Materiales y Métodos}

\section{Materiales}

Almidón de mandioca comercial fue proporcionado por Aldema ${ }^{\circledR}$ (Misiones, Argentina), el agua ultra pura fue provista a partir de un equipo de intercambio iónico Milli-Q, (Millipore Corporation, Burlington, MA, USA). El anhídrido acético, ácido acético glacial, metanol y glicerol fueron de calidad analítica (Cicarelli ${ }^{\circledR}$ Reagent SA, Santa Fé, Argentina). Ácido metilsulfónico y cloroformo, fueron proporcionado por Merck ${ }^{\circledR}$ (Kenilworth, Nueva Jersey, USA), (2-dodecen-1-il) anhídrido succínico (DSA) fue proporcionado por Sigma Aldrich ${ }^{\circledR}$ (San Luis, Misuri, USA) y papel Kraft de $85 \mathrm{~g} / \mathrm{m}^{2}$ fue adquirido comercialmente y utilizado en las condiciones de recepción.

\section{Síntesis de acetato de almidón de mandioca (AAM) alto grado de sustitución}

Para la síntesis de acetato de almidón de mandioca altamente sustituido, se utilizó el método de Feuer modificado [13] que propone una reacción en medio ácido con ácido metilsulfónico como catalizador.

El almidón, cuyo porcentaje de humedad es aproximadamente del $11 \%$, se secó previamente en estufa a $60^{\circ} \mathrm{C}$ durante toda la noche. Se mezclaron $30 \mathrm{~g}$ de almidón con $54 \mathrm{~mL}$ de anhídrido acético y $55 \mathrm{~mL}$ de ácido acético glacial. Luego, el catalizador de esterificación, ácido metilsulfónico (MSA), diluido en ácido acético, se añadió lentamente durante un período de 10 minutos. Posteriormente la mezcla se calentó y se mantuvo entre $80^{\circ} \mathrm{C}$ y $95^{\circ} \mathrm{C}$ en 120 minutos.

Al final, se añadió agua destilada fría $\left(5-6^{\circ} \mathrm{C}\right)$ a la solución para detener la reacción y precipitar el acetato de almidón. El precipitado blanco obtenido se lavó 2 veces con agua destilada y una con metanol, se secó en un horno a $40^{\circ} \mathrm{C}$ durante 24 horas y se almacenó en un desecador a temperatura ambiente.

\section{Determinación del grado de sustitución mediante valoración química por retorno}

El grado de sustitución del acetato de almidón se determinó mediante titulación de retorno utilizando el método de Miladinov \& Hanna [21]. Se colocó una cantidad de $0,5 \mathrm{~g}$ de almidón acetilado en un recipiente de $250 \mathrm{~mL}$ con agua destilada $(50 \mathrm{~mL})$ y se ajustó el $\mathrm{pH}$ a 7 con $\mathrm{HCl} 0,02$ $\mathrm{N}$. Luego se agregaron $25 \mathrm{~mL}$ de $\mathrm{NaOH} 0,5 \mathrm{~N}$ y se calen- 
taron en una placa caliente con agitación vigorosa hasta obtener una solución transparente. El exceso de $\mathrm{NaOH}$ se valoró de nuevo a pH 7 con $\mathrm{HCl}$. El grado de sustitución (DS) se calculó según lo propuesto por Xu y col. [12]:

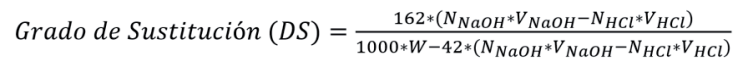

donde $\mathrm{NNaOH}$ es la normalidad de $\mathrm{NaOH}, \mathrm{VNaOH}$ es el volumen de $\mathrm{NaOH}, \mathrm{NHCl}$ es la normalidad de $\mathrm{HCl}$ usado para valorar de nuevo, $\mathrm{VHCl}$ es el volumen de $\mathrm{HCl}$ usado para titular.

\section{Preparación de películas de AAM}

Las películas fueron preparadas utilizando una concentración de acetato de almidón de mandioca (DS = $2,9)$ del 5\% (p/v) en cloroformo, empleando DSA como plastificante externo en las siguientes concentraciones: 0 , 20 y $50 \%$ (el porcentaje de plastificante está referido a la masa de AAM en la solución). Una vez homogeneizados los componentes, se introdujo un tiempo de espera de 4 hs a las soluciones filmogénicas para favorecer la plastificación del polímero antes del moldeo. Las películas libres se formaron por "casting" volcando $60 \mathrm{~mL}$ de la solución filmogénica en moldes de $10 \mathrm{~cm}$ de diámetro a $25^{\circ} \mathrm{C}$ y dejando evaporar el solvente por $24 \mathrm{hs}$. Una vez desmoldadas, las películas se almacenan en ambiente de humedad controlada $53 \%$.

\section{Preparación de materiales compuestos}

Para la preparación de la resina se solubilizó acetato de almidón de mandioca en cloroformo en concentraciones del 5, 10 y 20\% (p/v). Se ensayaron dos plastificantes externos de naturaleza química distinta DSA y glicerol en concentraciones del 5, 10 y 30\% referido a la masa de AAM en la solución. El refuerzo utilizado para el material compuesto fue papel Kraft, de $85 \mathrm{~g}$ cortado en discos de $12 \mathrm{~cm}$ de diámetro y pre secados en estufa a $40^{\circ} \mathrm{C}$ durante toda la noche. Los refuerzos fueron inmersos en la resina en diferentes tiempos (10, 30 y $50 \mathrm{~min})$. Los ensayos se realizaron según el diseño experimental presentado en la Tabla 1. Después de la impregnación las muestras fueron colocadas en desecador por 24 horas.

Tabla 1: Concentraciones y tiempo de inmersión utilizados en los ensayos iniciales de impregnación.

\begin{tabular}{|c|c|}
\hline Tiempo de inmersión (min) & Concentración de sol. AAM (\%) \\
\hline 10 & 5 \\
\hline 10 & 20 \\
\hline 50 & 5 \\
\hline 50 & 20 \\
\hline 30 & 10 \\
\hline 30 & 10 \\
\hline 50 & 10 \\
\hline
\end{tabular}

\section{Observación y micrografías}

Las observaciones microscópicas se realizaron, con un microscopio óptico marca Zeiss en un aumento de 100X, y se fotografiaron con cámara complementaria.

\section{Propiedades mecánicas}

Los ensayos de tracción se realizaron con una máquina de tensión / compresión marca Adamel Lhomagry (Francia) modelo DY 32 / 34 con rango de $10 \mathrm{~N}$ a $5 \mathrm{KN}$, con celdas intercambiables con una exactitud de $0,5 \%(0,1 \%$ a fondo de escala), rango de velocidades de 0,01 a $999 \mathrm{~mm} /$ min y con registro automático de tensión - deformación.

Se utilizaron probetas de $25 \mathrm{~mm}$ x $50 \mathrm{~mm}$ para películas libres y de $15 \mathrm{~mm}$ x $70 \mathrm{~mm}$ para materiales compuestos, registrándose tracción - deformación y energía absorbida a una velocidad constante de $0,4 \mathrm{~mm} / \mathrm{min}$.

\section{Permeabilidad al vapor de agua}

La velocidad de transmisión de vapor de agua (WVTR) de las películas libres y de los “composites" fueron evaluadas con el método cuasi-isostático, utilizado en envases de yerba [22] y siguiendo el procedimiento general descripto en las Normas TAPPI, T464 om-95 [23] y norma ASTM E96 [24].

Las experiencias se llevaron a cabo por triplicado. La humedad relativa externa (55\%) se fijó empleando solución salina saturada y se utilizó silica gel como desecante. La temperatura de las cabinas de ensayo se mantuvo constante en estufa con una precisión de $\pm 1^{\circ} \mathrm{C}$. Se registró la variación de peso de la celda en balanza electrónica marca Ohaus, con precisión de $0,001 \mathrm{~g}$.

\section{Resultados y Discusión}

\section{Acetato de almidón de mandioca}

Empleando el método de Feuer, se obtuvieron acetatos de almidón de mandioca (AAM) altamente hidrófobos, con un rendimiento de reacción del $85 \%$. El valor promedio de DS medidos por titulación para tiempo de reacción

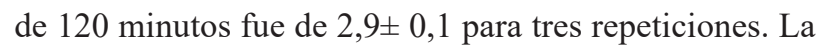
utilización de MSA como catalizador de reacción generó productos de mayor blancura y mayor peso molecular que otros métodos de acetilación, en coincidencia a los resultados de Feuer [13].

\section{Apariencia de películas de AAM}

Las películas obtenidas a partir de soluciones de acetato de almidón de mandioca (DS 2,9) resultaron homogéneas y continuas con respecto a su contenido y espesor, de apariencia plástica, pero muy frágiles. El 
agregado de plastificante a las soluciones filmogénicas mejoró la apariencia y las propiedades mecánicas (tensión y deformación de rotura) de los films obtenidos. Por otra parte, el hecho de que las películas sean completamente transparentes confirma la idea de que tanto el solvente como el plastificante fueron perfectamente compatibles en la matriz polimérica. Las películas de acetato de alto grado y $50 \%$ de DSA, mostraron excelentes propiedades al desmoldarlas (Figura 1a), pero luego de dos semanas de almacenamiento, se volvieron cerosas producto de la segregación del plastificante hacia la superficie de las mismas (Figura $1 \mathrm{~b}$ ), tornando a la película excesivamente quebradiza. Al ser el plastificante una molécula de alto peso molecular, de naturaleza mayormente apolar debido a su larga cadena hidrocarbonada, la asociación a las moléculas del polímero sería mayoritariamente debido a fuerzas de Van der Waals y al encontrarse en altas concentraciones en la matriz polimérica, la afinidad química hace que las moléculas migren hacia regiones de menor energía libre, esto es hacia la superficie. Por otro lado, la hidrólisis del anhídrido succínico (extremo polar) afectaría el estado de agregación del plastificante favoreciendo la segregación del mismo.

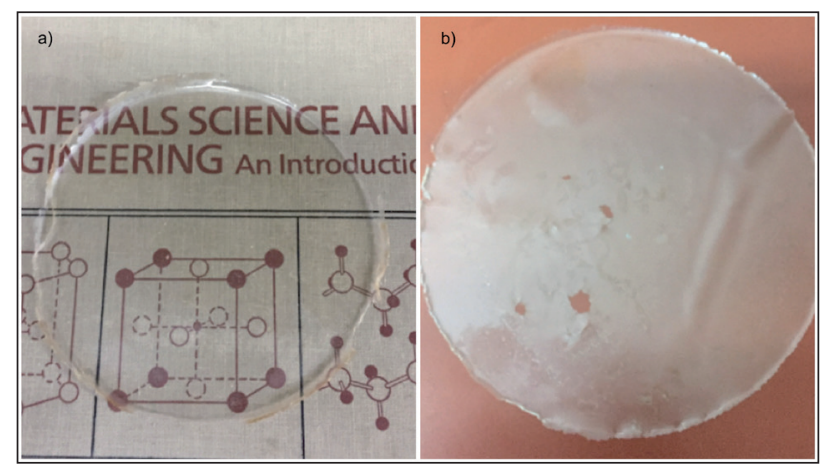

Figura 1: Películas de acetato de almidón de mandioca DS 2.9 al 5\% con $50 \%$ de DSA. a) recién desmoldada b) luego de dos semanas de almacenamiento.

\section{Permeabilidad al vapor de agua de películas de AAM}

En la Tabla 2 se presentan las propiedades de barrera al vapor de agua de la única formulación que resultó apta para el ensayo. Se encuentra una mejora significativa en las propiedades de barrera al vapor de agua de las películas de AAM obtenidas en este trabajo con respecto a las películas de almidón nativo de mandioca reportados por Mali y col. [25] sorbitol, glycerol-sorbitol mixture, urea and sucrose as plasticizers. The effects of these plasticizers on the microstructure, moisture sorption, water vapor permeability (WVP, Müller y col. [26] y de almidón nativo maíz informados por Slavutsky y Bertuzzi [27]. La baja permeabilidad medida podría atribuirse a la hidrofobicidad de la molécula de acetato de almidón, ya que los tres grupos hidroxilos han sido sustituidos en las tres posiciones posibles de la anhidroglucosa y los grupos acetato sustituyentes dotan de carácter apolar a las moléculas del polímero [5]. Por otro lado, al utilizarse un plastificante de la misma naturaleza química, se mantiene el efecto de la hidrofobicidad del film al tiempo que se evitan fenómenos de incompatibilidad que normalmente derivan en agrietamientos.

Tarvainen y col. [18] potato starch acetate degree of substitution 2.8 (SA reportan para almidones de papa con DS 2,8 con $50 \%$ de anhídrido octenil succínico (OSA) como plastificante, valores de permeabilidad de 1,63.10$10 \mathrm{~g} / \mathrm{msPa}$, semejantes que los obtenidos en el presente trabajo.

\section{Propiedades mecánicas de películas de AAM}

Las películas no plastificadas y con contenidos de DSA del $20 \%$ desarrolladas en este estudio fueron demasiado rígidas y frágiles, por lo que se requirió un contenido de plastificante del 50\% para lograr películas aptas para las pruebas de tracción. La alta concentración de plastificante requerida para relajar las cadenas del polímero, podría deberse a la estructura molecular compleja del AAM y a su alto peso molecular. Tarvanein y col. [17] detallan que la estructura compleja del acetato de almidón crea lugares de enlaces para cadenas de polímero adyacentes, lo que permite que se formen fuertes fuerzas cohesivas y estructuras tenaces y densas en los films. En la Figura 2 se presenta la curva característica tensión - deformación de películas de AAM con 50\% de DSA. Las propiedades mecánicas de estas películas, demuestran que el material es dúctil con valores de tensión de rotura promedio 17,50 $\pm 0,60 \mathrm{MPa}$ y deformación de rotura de $8,24 \pm 0,70 \%$. Los valores máximos de tensión obtenidos en este estudio fueron significativamente mejores que los reportados por Tarvanein y col. [18]potato starch acetate degree of substitution 2.8 (SA (9,1 Mpa) para películas de acetato de almidón de papa con el mismo DS utilizando el mismo plastificante.

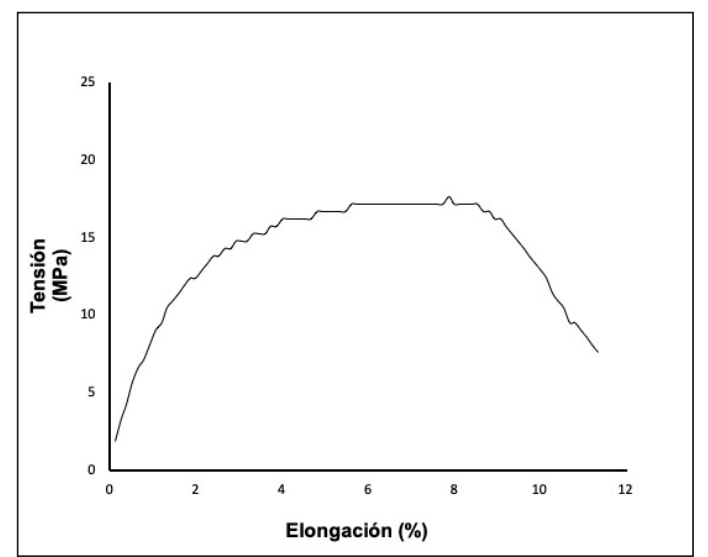

Figura 2: Diagrama Tensión - Elongación de películas de acetato de almidón de mandioca con grado de sustitución de 2,9 y 50\% de DSA como plastificante. 


\section{Materiales compuestos: apariencia y micrografías de las películas compuestas}

La apariencia de los papeles impregnados con la resina de AAM, se asemejó a un papel encerado o al de una película "plástica", traslúcida, esto fue especialmente notable en los ensayos de mayores tiempos de inmersión y concentraciones. Las micrografías que muestran el efecto del tiempo de inmersión en soluciones de AAM a distintas concentraciones, así como el efecto del agregado de plastificantes de distinta naturaleza química se presentan en la Figura 3.

En la micrografía del papel sin tratar (blanco), se aprecian las fibras del papel que con la impregnación en acetato de almidón dejaron de ser visibles, esto se debió a la incorporación de acetato de almidón en los espacios entre fibras. Este efecto se incrementó conforme aumentó el tiempo de inmersión para materiales con resinas del 10\% de AAM, sugiriendo que el "coating" se produce por la incorporación de la resina al interior del "film" en lugar de ser un fenómeno netamente superficial.

Para concentraciones del 20\% de AAM, aún en tiempos cortos de inmersión (10 min), se observó un "cuarteado", en la superficie del material. Esta falta de homogeneidad se podría deber a que a esta concentración la cantidad de resina que permanece sobre la superficie forma una capa de mayor espesor, la cual en el proceso de secado sufre agrietamiento.

Los plastificantes ensayados no formaron una fase continua en el material, sino que se visualizó segregación en la superficie, tanto para el caso del glicerol como para el DSA (Figura 3e y 3f).

\section{Permeabilidad al vapor de agua de materiales compuestos}

En la Figura 4 se presentan los resultados de ganancia de peso y VWTR en celdas cubiertas con papeles Kraft sin tratar e impregnados en soluciones de acetato de almidón
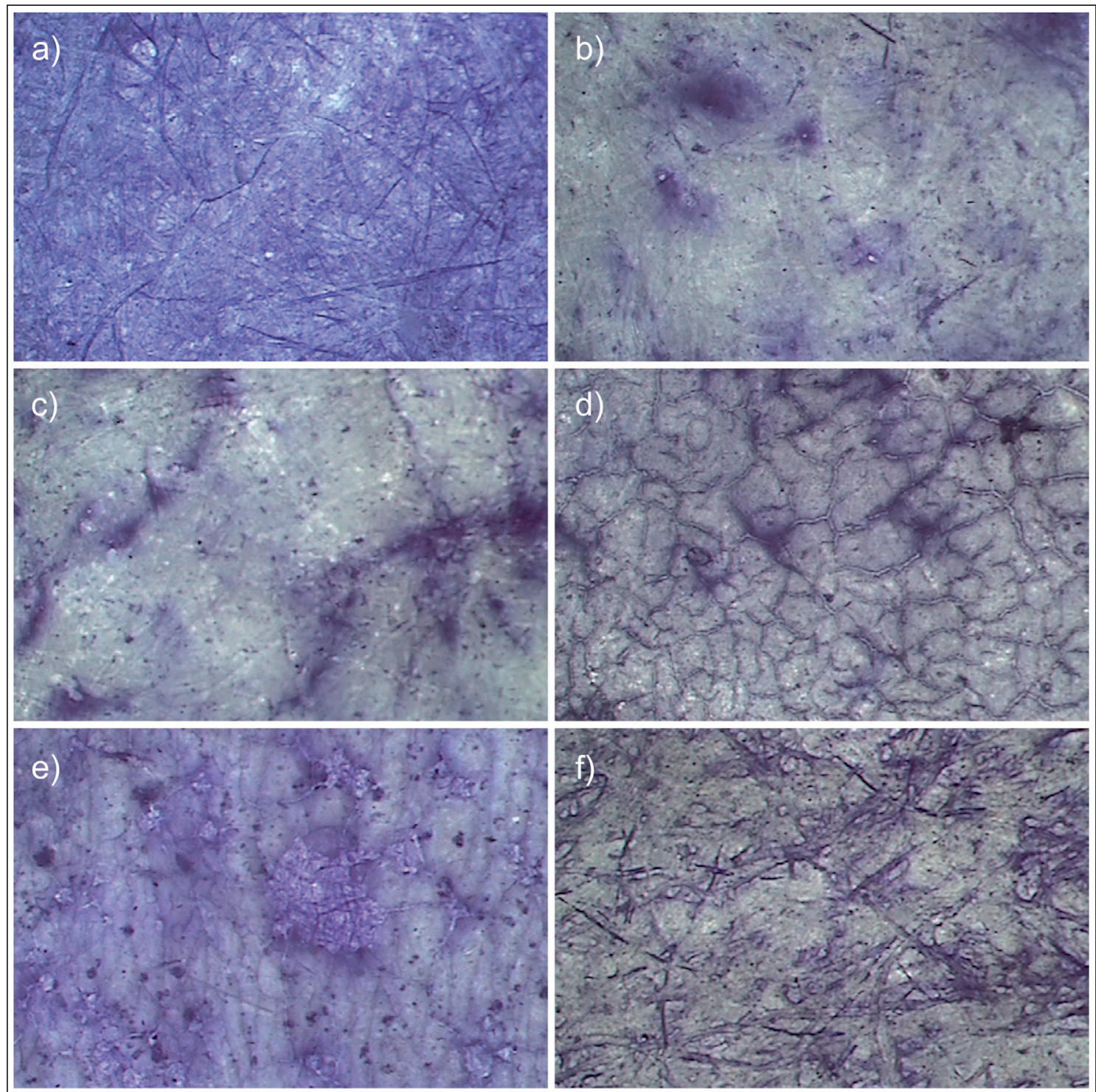

Figura 3: Micrografías de: a) Papel Kraft sin tratar. b) 10\% de AAM - 10 min de inmersión. c) 10\% AAM - 50 min de inmersión. d) 20\% de AAM - 10 min de inmersión. e) 10\% de AAM - 30\% glicerol - 30 min de inmersión. f) 10\% de AAM - 5\% de DSA - 10 min de inmersión. 
de mandioca de concentraciones de 5, 10 y $20 \%$, utilizando tiempos de impregnación de 10 minutos, expuestas a condiciones ambientales $55 \% \mathrm{HR}$ y $38^{\circ} \mathrm{C}$.
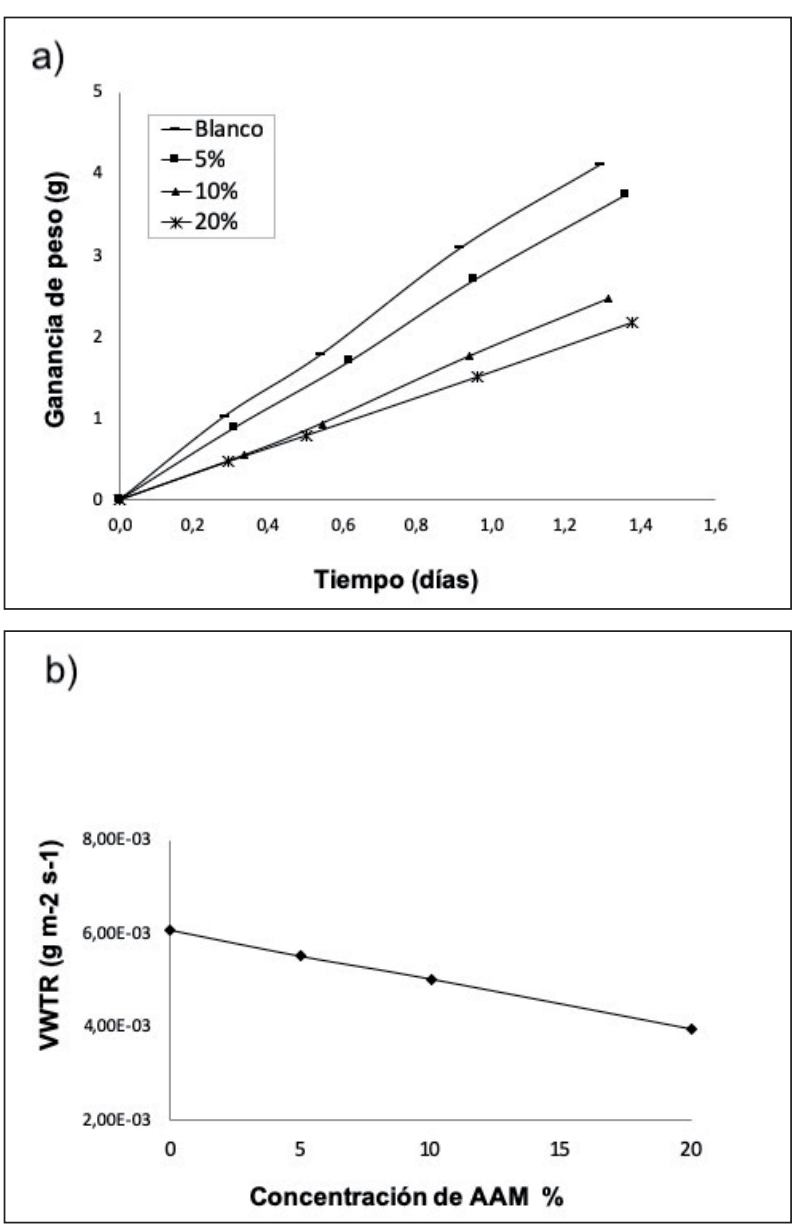

Figura 4: Valores de (a) Ganancia de peso y (b) VWTR de papeles impregnados en AAM 5,10 y $20 \%$, medidos a $38^{\circ} \mathrm{C}$, 0 y $55 \% \mathrm{HR}$.

Desde el comienzo las celdas cerradas con papeles impregnados ganaron menos agua, lo que implicó menor permeabilidad al vapor de agua y esta mejora fue proporcional a la concentración de acetato en el rango de concentraciones estudiado.

Los valores de velocidad de transmisión al vapor de agua (VWTR) y permeabilidad (P) calculados en papeles impregnados durante 10 minutos, expuestos a condiciones ambientales $55 \% \mathrm{HR}$ y $38^{\circ} \mathrm{C}$, para las distintas concentraciones de AAM ensayadas, se presentan en la Tabla 2.

Tabla 2: Valores de VWTR y permeabilidad calculados para películas y papeles impregnados en distintas concentraciones de AAM y expuestos a $55 \% \mathrm{HR}$ a $38^{\circ} \mathrm{C}$

\begin{tabular}{|c|c|c|c|}
\hline \multirow{2}{*}{ Material } & VWTR & Desviación & Permeabilidad \\
\cline { 2 - 4 } & $\mathbf{( g / \mathbf { m } ^ { 2 } \mathbf { s } )}$ & Estándar VWTR & $\mathbf{( g / m s P a )}$ \\
\hline AAM 5\% 50\% DSA * & $2,04.10^{-03}$ & $2,91.10^{-5}$ & $1,29.10^{-10}$ \\
\hline Papel Kraft+ 0\% AAM & $6,90.10^{-3}$ & $4,71.10^{-4}$ & $2,52.10^{-10}$ \\
\hline Papel Kraft+ 5\% AAM & $5,53.10^{-3}$ & $3,88.10^{-4}$ & $2,02 \cdot 10^{-10}$ \\
\hline Papel Kraft+ 10\% AAM & $5,04.10^{-3}$ & $2,12.10^{-4}$ & $1,84.10^{-10}$ \\
\hline Papel Kraft+20\% AAM & $3,97.10^{-3}$ & $4,95.10^{-5}$ & $1,45 \cdot 10^{-10}$ \\
\hline
\end{tabular}

*El espesor promedio de las películas es de 1,5.10-4m.
Puede observarse que se encuentra una mejora del 58\% en las propiedades barrera del papel cuando se aumenta la concentración de las soluciones de impregnación al 20\% de AAM, para un tiempo de inmersión de 10 minutos. Larotonda y col. [19], obtuvieron reducciones de permeabilidad del mismo orden para resinas compuestas de un $20 \%$ de AAM con un DS de 1,41. Teniendo en cuenta que en este trabajo el grado de sustitución del almidón acetilado fue de 2,9, no pareciera haber un efecto del grado de sustitución del AAM en este rango.

Por otra parte, cuando se estudió el efecto del tiempo de inmersión para soluciones de AAM en cloroformo en concentraciones de 10 y $20 \%$, la permeabilidad al vapor de agua disminuyó con el aumento del tiempo de inmersión de manera significativa. Los resultados pueden verse en la Figura 5.

Con soluciones al 10\% se observó una disminución regular del valor de permeabilidad al vapor de agua, expresado como VWTR, para todos los tiempos de tratamiento en la solución de AAM. La permeabilidad alcanzó a un $74 \%$ del valor de permeabilidad del papel sin tratar para un tiempo de inmersión de 10 min y llegó solo al 51\% para 50 minutos de inmersión.

Cuando los ensayos para estudiar el efecto del tiempo de impregnación se realizaron con soluciones de AAM en cloroformo al 20\%, los valores de VWTR medidos presentaron una significativa disminución del $40 \%$ en los primeros tiempos de tratamiento (10 min). Fringant y col. [28], estudiaron el efecto de sucesivas aplicaciones de acetato de almidón de trigo de DS 2,8 en diclorometano sobre papel, encontrando una mejora significativa en la resistencia al agua líquida (similar al polietileno) y proporcional al espesor del film, sin embargo, no encontraron una mejora significativa respecto a la permeabilidad al vapor de agua. La poca eficiencia del triacetato de almidón para reducir la permeabilidad, encontrada por Fringant y col. [28], a diferencia de este estudio, se debería fundamentalmente al modo de aplicación del "coating", ya que en el presente trabajo la incorporación de la resina se da en toda la matriz del papel, como se infiere a partir del efecto del tiempo de inmersión que indica un efecto de la difusión de la resina en la matriz del papel.

En cuanto al agregado de plastificantes a las resinas, no se encontró un efecto significativo de mejora en las propiedades de barrera al vapor de agua, este evento es esperable ya que en las micrografías no se observó la formación fases continuas sobre la superficie del film, en las que el plastificante se agrupó formando micelas.

\section{Propiedades mecánicas de materiales compuestos}

Los papeles impregnados en solución de AAM al 10 y al 20\%, utilizando tiempos de inmersión de 10, 30 y 50 minutos fueron sometidos a ensayos de tracción. Los resultados promedio de 4 repeticiones son mostrados en 


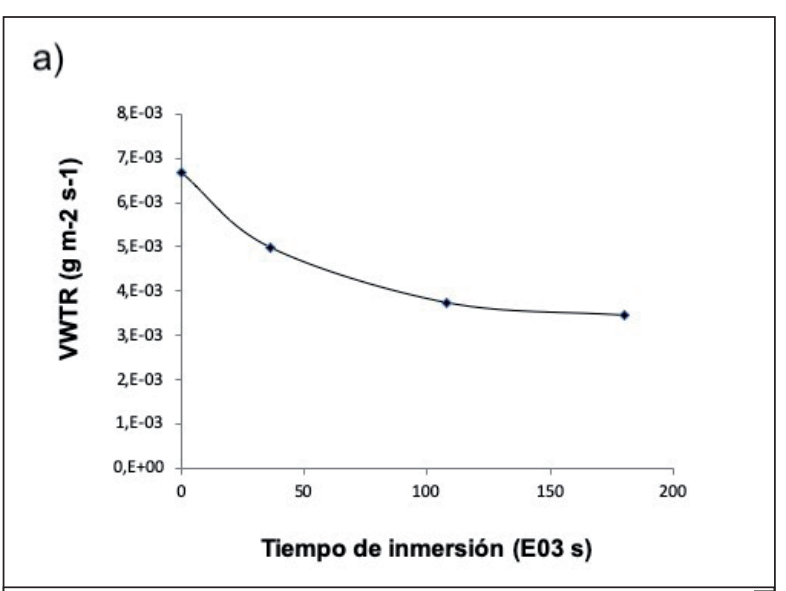

b)

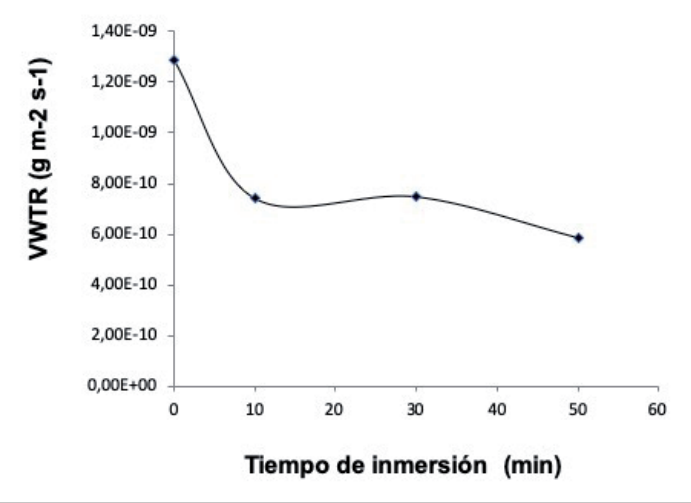

Figura 5: Efecto del tiempo de inmersión en soluciones de (a) AAM al $10 \%$ y (b) AAM al $20 \%$.

\section{la Tabla 3.}

Tabla 3: Valores de tracción, elongación y energía de rotura para papeles con distintos tiempos de impregnación, con resinas al 10 y $20 \%$ de AAM.

\begin{tabular}{|c|c|c|c|c|}
\hline \multirow{3}{*}{ Muestra } & Tiempo & Tracción & Elongación & Energía \\
\cline { 2 - 5 } & $\min$ & $\boldsymbol{\sigma}(\mathbf{M P a})$ & $(\%)$ & $(\mathbf{m J})$ \\
\hline \multirow{3}{*}{$\begin{array}{c}10 \% \text { de } \\
\text { AAM }\end{array}$} & 0 & $19,55 \pm 1,81^{\mathrm{a}}$ & $2,18 \pm 0,13^{\mathrm{a}}$ & $37,16 \pm 4,07$ \\
\cline { 2 - 5 } & 10 & $35,47 \pm 3,06^{\mathrm{b}}$ & $2,50 \pm 0,21^{\mathrm{ab}}$ & $84,00 \pm 14,14$ \\
\cline { 2 - 5 } & 30 & $33,92 \pm 1,80^{\mathrm{b}}$ & $2,12 \pm 0,16^{\mathrm{ab}}$ & $64,23 \pm 2,03$ \\
\hline \multirow{3}{*}{$\begin{array}{c}20 \% \text { de } \\
\text { AAM }\end{array}$} & 50 & $33,12 \pm 2,90^{\mathrm{b}}$ & $3,34 \pm 0,32^{\mathrm{b}}$ & $105,55 \pm 0,32$ \\
\cline { 2 - 5 } & 0 & $19,55 \pm 1,82^{\mathrm{a}}$ & $2,18 \pm 0,13^{\mathrm{a}}$ & $37,16 \pm 4,07$ \\
\cline { 2 - 5 } & 30 & $33,94 \pm 2,01^{\mathrm{b}}$ & $4,12 \pm 0,38^{\mathrm{ab}}$ & $139,14 \pm 16,92$ \\
\cline { 2 - 5 } & 50 & $35,85 \pm 1,66^{\mathrm{b}}$ & $3,79 \pm 0,60^{\mathrm{ab}}$ & $128,01 \pm 27,06$ \\
\hline
\end{tabular}

Diferentes letras en la misma columna indican diferencias estadísticas significativas para un nivel de confianza del $95 \%$.

Los valores de tracción de rotura demuestran una mejora significativa en los papeles impregnados con respecto a los papeles sin tratar. La tendencia se apreció claramente en los ensayos realizados para resinas del 20\% de AAM. Los resultados obtenidos para el 10\% de AAM presentaron cierta dispersión con respecto al tiempo de inmersión, pero en todos los casos se obtuvo una mejora en las propiedades mecánicas. Los valores de energía consumida hasta la rotura también mostraron un aumento con el tratamiento de impregnación y estuvieron en un rango del 72 al 87\% mayor que el valor de la muestra sin tratar.

\section{Conclusiones}

Fue posible sintetizar acetato de almidón de mandioca de alto grado de sustitución (DS 2,9), insoluble en agua y con buen rendimiento de reacción. A partir del ester de almidón, se desarrollaron películas libres y materiales compuestos consistentes en una resina del polímero sintetizado, soportado en papel Kraft.

Las películas de almidón de mandioca acetilado resultaron homogéneas y continuas, de apariencia plástica, pero muy frágiles. El agregado 50\% de DSA permitió obtener películas con buenas propiedades mecánicas (tensión de rotura $17,5 \mathrm{MPa}$, deformación $8,2 \%$ ) pero que con el almacenamiento, se volvieron cerosas y frágiles.

Los materiales compuestos de papel Kraft - acetato de almidón de mandioca $\mathrm{DS}=2,9$ tienen valores de permeabilidad al vapor de agua menores (hasta el 51\%) que las de los papeles sin resina, a la vez que sus propiedades mecánicas son mejores (tracción y energía de rotura $72-87 \%$ mayores). Hay un efecto positivo de la concentración de acetato de la solución impregnante y del tiempo de inmersión sobre los valores de VWTR. El efecto del tiempo de inmersión y las micrografías sugieren que la incorporación de acetato ocurre "en masa" es decir incorporándose en los espacios interfibra de la estructura del papel Kraf, y no formando únicamente una película superficial.

Este estudio demuestra que la utilización de acetato de almidón de alto grado de sustitución en el desarrollo de materiales compuestos sería una alternativa interesante en la generacion de envases biodegradables.

\section{Referencias}

1. A.M. Youssef, F.M. Assem, S.M. El-Sayed, H. Salama, M.H. $A b d$ El-Salam, Utilization of Edible Films and Coatings as Packaging Materials for Preservation of Cheeses, J. Packag. Technol. Res. 1 (2017) 87-99. doi:10.1007/ s41783-017-0012-3.

2. P.G. Seligra, C. Medina Jaramillo, L. Famá, s. Goyanes. Biodegradable and non-retrogradable eco-films based on starch-glycerol with citric acid as crosslinking agent, Carbohydr. Polym. 138 (2016) 66-74. doi:10.1016/j. carbpol.2015.11.041.

3. T. Garrido, M. Peñalba, K. de la Caba, P. Guerrero. A more efficient process to develop protein films derived from agro-industrial by-products, Food Hydrocoll. 86 (2019) 11-17. doi:10.1016/j.foodhyd.2017.11.023.

4. X. Jiang, H. Li, Y. Luo, Y. Zhao, L. Hou. Studies of the plasticizing effect of different hydrophilic inorganic salts on starch/ poly (vinyl alcohol) films, Int. J. Biol. Macromol. 82 (2016) 223-230. doi:10.1016/j.ijbiomac.2015.11.046.

5. P. Cuenca, S. Ferrero, o. Albani. Preparation and charac- 
terization of cassava starch acetate with high substitution degree, Food Hydrocoll. 100 (2020) 105430. doi:10.1016/j.foodhyd.2019.105430.

6. J. Singh, L. Kaur, N. Singh. Effect of acetylation on some properties of corn and potato starches, Starch/Staerke. (2004). doi:10.1002/star.200400293.

7. N.S. Sodhi, N. Singh. Characteristics of acetylated starches prepared using starches separated from different rice cultivars, J. Food Eng. (2005). doi:10.1016/j.jfoodeng. 2004.09.018.

8. O. V. López, N.E. Zaritzky, M.A. García. Physicochemical characterization of chemically modified corn starches related to rheological behavior, retrogradation and film forming capacity, J. Food Eng. 100 (2010) 160-168. doi:10.1016/j.jfoodeng.2010.03.041.

9. R. Colussi, V.Z. Pinto, S.L.M. El Halal, N.L. Vanier, F.A. Villanova, R. Marques E Silva, E. Da Rosa Zavareze, A.R.G. Dias. Structural, morphological, and physicochemical properties of acetylated high-, medium-, and low-amylose rice starches, Carbohydr. Polym. (2014). doi:10.1016/j. carbpol.2013.12.070.

10. O.F. Osundahunsi, K.T. Seidu, R. Mueller. Effect of presence of sulphurdioxide on acetylation and sorption isotherm of acetylated starches from cultivars of cassava, Food Chem. 151 (2014) 168-174. doi:10.1016/j.foodchem.2013.11.020.

11. R.L. Shogren. Rapid preparation of starch esters by high temperature/pressure reaction, Carbohydr. Polym. (2003). doi:10.1016/S0144-8617(02)00305-3.

12. Y. Xu, V. Miladinov, M.A. Hanna. This article is from the November-December 2004 issue of published by the American Association of Cereal Chemists, Inc . Synthesis and Characterization of Starch Acetates with High Substitution 1, Cereal Chem. 81 (2004) 735-740.

13. B.I. Feuer. Method of making starch acetate using an acid catalyst, 5710269, 1998.

14. C.I.K. Diop, H.L. Li, B.J. Xie, J. Shi. Effects of acetic acid/ acetic anhydride ratios on the properties of corn starch acetates, Food Chem. 126 (2011) 1662-1669. doi:10.1016/j.foodchem.2010.12.050.

15. C. Fringant, M. Rinaudo, M.F. Foray, M. Bardet. Preparation of mixed esters of starch or use of an external plasticizer: Two different ways to change the properties of starch acetate films, Carbohydr. Polym. 35 (1998) 97-106. doi:10.1016/S0144-8617(97)00250-6.

16. J. Guan, M.A. Hanna. Extruding foams from corn starch acetate and native corn starch, Biomacromolecules. (2004). doi:10.1021/bm049512m.
17. M. Tarvainen, R. Sutinen, S. Peltonen, P. Tiihonen, P. Paronen. Starch acetate - A novel film-forming polymer for pharmaceutical coatings, J. Pharm. Sci. 91 (2002) 282-289. doi:10.1002/jps.10027.

18. M. Tarvainen, R. Sutinen, S. Peltonen, H. Mikkonen, J. Maunus, $\mathrm{K}$. Vähä-Heikkilä, V.P. Lehto, P. Paronen. Enhanced film-forming properties for ethyl cellulose and starch acetate using n-alkenyl succinic anhydrides as novel plasticizers, Eur. J. Pharm. Sci. 19 (2003) 363-371. doi:10.1016/ S0928-0987(03)00137-4.

19. F.D.S. Larotonda, K.S. Matsui, S.S. Paes, J.B. Laurindo. Impregnation of Kraft paper with cassava-starch acetateAnalysis of the tensile strength, water absorption and water vapor permeability, Starch/Staerke. 55 (2003) 504-510. doi:10.1002/star.200300179.

20. F.D.S. Larotonda, K.N. Matsui, P.J.A. Sobral, J.B. Laurindo. $H y$ groscopicity and water vapor permeability of Kraft paper impregnated with starch acetate, J. Food Eng. 71 (2005) 394-402. doi:10.1016/j.jfoodeng.2004.11.002.

21. V.D. Miladinov, M.A. Hanna. Starch esterification by reactive extrusion, Ind. Crops Prod. (2000). doi:10.1016/ S0926-6690(99)00033-3.

22. L.A. Ramallo, O.A. Albani. Prediction and Determination of Water Uptake in Packaged Yerba Mate, Food Sci. Technol. Int. 10 (2004) 35-40. doi:10.1177/1082013204041877.

23. J. Trotter. Standard conditioning and testing atmospheres for paper, board, pulp handsheets, and related products (Revision of T 402 sp-08), 2013.

24. c. Methods. Standard Test Methods for Water Vapor Transmission of Shipping Containers -, 2003. doi:10.1520/D4279-95R09.2.

25. M.C. Galdeano, S. Mali, M.V.E. Grossmann, F. Yamashita, M.A. García. Effects of plasticizers on the properties of oat starch films, Mater. Sci. Eng. C. 29 (2009) 532-538. doi:10.1016/j.msec.2008.09.034.

26. L.J.Y. Muller M, Muller. Cellulose reinforced starch .pdf, Food Hydrocoll. 23 (2009) 1328-1333.

27. A.M. Slavutsky, M.A. Bertuzzi. Water barrier properties of starch films reinforced with cellulose nanocrystals obtained from sugarcane bagasse, Carbohydr. Polym. 110 (2014) 53-61. doi:10.1016/j.carbpol.2014.03.049.

28. C. Fringant, M. Rinaudo, N. Gontard, s. Guilbert, H. Derradji, A Biogradable Starch Based Coating to Waterproof Hydrophilic Materials, Starch - Stärke. 50 (1998) 292-296. doi:10.1002/(sici)1521379x(199807)50:7<292::aid-star292>3.3.co;2-r. 